\title{
Detection of Traffic Light using Machine Vision for Autonomous Vehicles Application
}

\author{
Nur Hanim Suraya Bt. Sabarudin, MohdFauzi Bin Alias, Mohamed Yusof Bin Radzak
}

\begin{abstract}
Traffic light detection is crucial to decrease the traffic light accidents at intersections and to realize autonomous driving. There are so many existing methods to detect traffic light. However, these approaches have several limitations, such as not function well in complex driving environments. Hence, to overcome such constraints, the traffic light detection for the autonomous vehicle using image processing technique is proposed. The experiments are carried out using 114 scene images that consist of 209 traffic lights with different angles, weather conditions, and distance. An image processing technique, Hough Circle Transform is used in this traffic light detection system with the help of Gaussian blurring and Sobel filter. So, the overall accuracy rate for the proposed algorithm is $75.59 \%$. This system is possible to be used in urban areas or complex environments, whether it is at night or day, and it able to detect the traffic light regardless of the colour changes.
\end{abstract}

Keywords: Autonomous vehicle, Hough circle transform, Image processing technique, Traffic light detection

\section{INTRODUCTION}

The published crash statistics show that 136 Malaysians are death and 155 injured in 2002 due to traffic light violation related crashes and the number of fatalities due to traffic light-related accidents has continuously increased, leading to 182 deaths and 757 injuries in 2009 [1]. Therefore, states of traffic lights are essential components of traffic environments. Knowing the rules of traffic lights allows the automotive navigation system to efficiently identify the best route to any destination and can avoid crashes at intersections. Unlike humans, the driverless vehicle does not have the eyes to see the actual road conditions where it only relies on numerous sensors to perceive the surroundings. Moreover, in urban areas have often complex background characteristics which can add to various sources of distractions for the autonomous vehicle to maneuver at the cross-section.

In this case, the proper detection of traffic lights and the perception of its status as a great significance to avoid

Revised Manuscript Received on December 30, 2019.

* Correspondence Author

Mohamed Yusof Bin Radzak, Electrical, Electronics and Automation Section, Malaysian Spanish Institute, Universiti Kuala Lumpur, Kulim Hi-Tech Park, Kedah, Malaysia. Email: myusofr@unikl.edu.my

Nur Hanim Suraya Bt. Sabarudin, Pursuing B.Eng Technology, Mechatronics (Automotive), Universiti Kuala Lumpur, Malaysia.

Mohd Fauzi Bin Alias, Lecturer, Electrical, Electronics and Automation Section, Universiti Kuala Lumpur, Malaysia.

(C) The Authors. Published by Blue Eyes Intelligence Engineering and Sciences Publication (BEIESP). This is an open access article under the CC BY-NC-ND license (http://creativecommons.org/licenses/by-nc-nd/4.0/) accidents at intersections and also can ensure safe navigation through the cross-section. Traffic light detection is an integral part of autonomous vehicle systems which provides timely and appropriate reactions to traffic lights in cross-sections.

Hence, the traffic light detection for the autonomous vehicle using the image processing technique has proposed.

This system uses scene images as input images. Then the traffic light detection system will read the image from memory and start processing. In this traffic light detection system, red will indicated that the vehicle should slow down even more or stop in front of the traffic light. Yellow will suggest that the car should slowdown and green is the vehicle that can pass.

\section{RELATED WORKS}

In realizing an autonomous vehicle, the traffic light detection and recognition system play an essential role to ensure timely and appropriate reactions to traffic lights in cross-sections. Believe it or not, in non-autonomous vehicles, traffic light state detection would also be useful because it will warn distracted drivers to be more alert against the change of light status and making intersections safer. Besides, the proper detection of traffic lights and the perception of its condition is a great significance to avoid accidents at intersections and also can ensure safe navigation through the cross-section.

Most studies on traffic light detection and recognition only began around the 2000s, unlike traffic sign detection, where the first research can be traced back to 1987 [2]. Among the early research on traffic light detection and recognition can be found in [3] where the researchers propose a method to detect the state of the traffic light by extracting candidates for the traffic light region with colour similarity and selecting a right traffic light by using affine moment invariants. Until today, there are countless research, studies and experiments conducted by researchers all over the world using different types of detection methods for the traffic lights detection system. There have been large numbers of research in the general problem of detection of a traffic light. Research done by [4], use a HUV- histogram to detect the traffic light. The discovery and extraction of the traffic light image patches from captured driving- view images by using aggregate channel features (ACF). After that, to recognize states of traffic lights, the researchers remove irrelevant background pixels in the detected traffic light image patches by using visual saliency analysis, then train support vector machine (SVM) with HUV-histogram. By using this algorithm, the traffic light recognition system can operate in Korean highways and urban areas during the day only. 


\section{Detection of Traffic Light Using Machine Vision for Autonomous Vehicles Application}

According to a research study by [5], the approach used relies on Euclidean distance transform (EDT) and local contour pattern (LCP) which use both colour and shape details to detect the traffic lights. First, it combines principle component analysis (PCA) with EDT-based image to recognize colour and perform a more detailed segmentation in colour space to boost the detection performance. Then, the researcher incorporated the above colour detection into the contour segmentation of traffic light holder based on the LCP to enhance the recognition rate of the traffic light. In this study, the researcher stated that this recognition approach could recognize traffic light that is far away from the camera.

After that, in [6], the researcher proposed traffic light detection on the image using the fully convolutional network (FCN) with clustering. The input FCN takes one colour image of the road scene, and at the output of the neural network, there is a single-channel grayscale map. Next, apply the empirically chosen brightness threshold at the binarization stage. Then, the DBSCAN algorithm needs to cluster the resulting binarized image into closed regions. Then the detected regions are filtered before discarded too small clusters with an area less than 30 pixels. The bounding boxes form around the remaining groups, which are the result of the traffic signal detection algorithm.

The method uses by [7] in their research is to recognize the traffic light based on Hough Transform since it can realize pattern and forms in an image like the circular shapes. In this study, the researcher includes pixel clustering, edge detection and voting as their algorithm where the parameters with the maximum vote are selected as the parameters of the turned-on light. In order to detect the colour of the turned-on and turned-off lights, the pixels are clustered by their colour. After that, to extract the turned-on light, the edges are detected from the image by using the Sobel filter. Finally, the circle with the maximum vote is selected as the detected traffic light. Overall, by using this algorithm, it can detect a traffic light in a reasonable time and have better accuracy.

The contribution by [8], presents a system composed of the pre-learned Haar-like feature and SVM classifier. The researcher uses Haar-like features to learn about the traffic light image and detect the candidate area based on the learning data. The identified candidate image then is verified by the pre-learned Support Vector Machine (SVM) classifier. The result of the proposed algorithms shows that it is possible to detect the traffic light regardless of the colour changes.

Next, in [9], the researcherstated that there are three stages in this research, where the first one is to extract the traffic light by using the colour threshold segmentation method. After that, the noise removal and filter are applied before the template matching; the normalized cross-correlation techniques are used. This work is claimed to be effective and robust to use in complex urban environments.

\section{METHODOLOGY}

There are two stages in this project which the first used to determine the threshold range values based on the traffic light colour recognition. After the threshold range values are determined, then the second stage is applied to detect and classify the traffic light in the scene images. Fig. 1 and Fig. 2 show the flowchart for both algorithms used in this research.

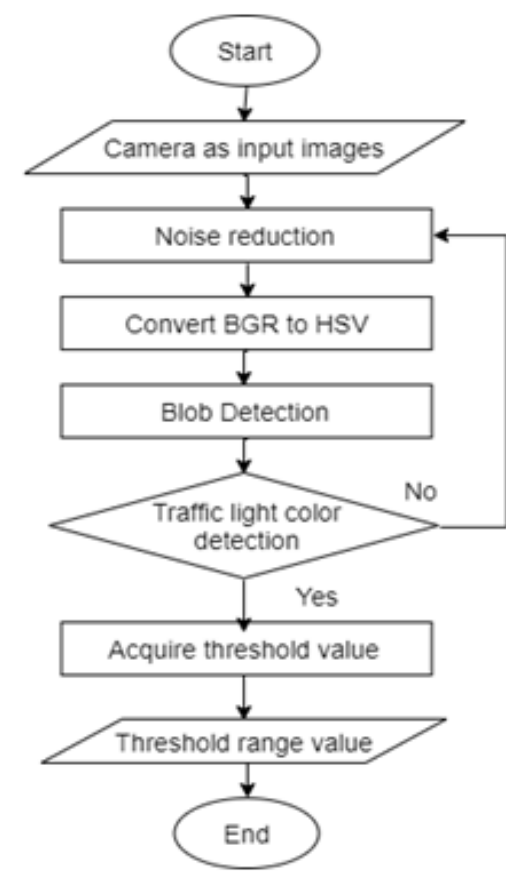

Fig. 1. Flowchart to Determine Threshold Range Value

\section{A. Determine the Threshold Range Value.}

As shown in Fig.1, a camera is used to gives the actual visual of the traffic light colour where it captures the images in real-time and saves them in the computer memory. Then the traffic light detection system will read the image from memory and start processing. The Gaussian Blur is applied to remove the noise in input images before the BGR colour-space is converted into HSV. Then, this detection system will use Blob Detection to detect blobs and filter it based on different characteristics. In this research, the HSV value is manually adjusted. So if there are any traffic light color that is detected, then the HSV value will be considered as acceptable, and the amount will be taken.

\section{i. Noise removal.}

Noise can be caused by many factors, including dirty lenses, faulty electronic components, low resolution or bad lighting. Hence, the image smoothing is required and can be achieved by convolving the image with a low-pass filter kernel which is very useful for eliminating high-frequency content like noise and edges from the image. In OpenCV library, there are four main types of blurring techniques which are averaging, Gaussian blurring, median blurring and bilateral filtering. However, in this algorithm, the Gaussian kernel is used since it is highly effective in removing Gaussian noise from the image.

\section{ii. Blob detection algorithm.}

The blob refers to a group of connected pixels in a binary image that shares some common property. The blob detection is based on an algorithm that is consists of thresholding, grouping, merging and also centre and radius calculation. First, the thresholding is applied to convert the input images to several binary images. After that, the binary images pixels which are white are grouped.

Published By:

Blue Eyes Intelligence Engineering 
Then, the centre of the blobs is calculated and merged. Lastly, the centre and radii of the newly merged blobs are computed and returned. However, this algorithm is controlled by several parameters. Hence, in this research, the blobs are filtered according to the area, circularity, and convexity.

\section{B. Algorithm to detect and classify the traffic light}

Based on Fig. 2, in this algorithm, the scene images will serve as the input images. Then, the image thresholding method is applied by manually set the HSV value in the program code by referring to the threshold range value that has been obtained before. A Gaussian blur is applied to reduce noises in input images. Next, to extract a colour input image, the colour conversion method is used where it converts from BGR image to HSV since it is easier to represent a traffic light colour in HSV than BGR colour-space. After that, a feature extraction technique which is Hough Circle Transform and Sobel filter are applied to the input images where it usually used to detect circles and edges in an image. The circle with a maximum vote is selected as the detected traffic light. Lastly, the detected traffic light is classified according to their class before the analysis for the system performance, and accuracy is made.

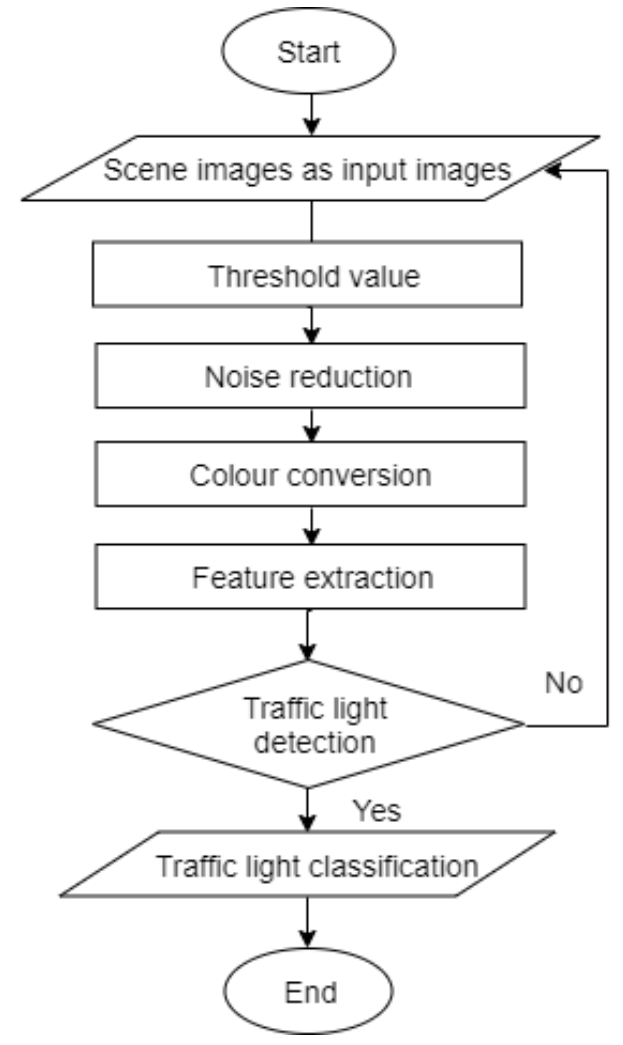

Fig. 2. Flowchart of Detection and Classification.

There are two feature extraction techniques used in this algorithm which are Hough Circle Transform and Sobel filter. The Hough Circle Transform technique generally used for detecting the circular objects in images with the goals to find the circles in imperfect image inputs. In this technique, the circles are produced by "voting" in the Hough parameter space and then select the local maxima in an accumulator matrix while Sobel filter is used in edge detection algorithms where it produces an image that emphasizes edges. Technically, it is a discrete operator of differentiation that computing an approximation for image intensity function gradient.

\section{IV.RESULTS AND DISCUSSIONS.}

Initial experiments on traffic lights detection tested and processed by using a Raspberry Pi board that have Ubuntu 16.04 LTS (Xenial Xerus) operating system. A camera used is Raspberry $\mathrm{Pi}$ camera module v2 because it provides a resolution of $3280 \times 2464$ pixels with a wide field of view $\left(62.2^{\circ} \times 48.8^{\circ}\right)$ that attached in front of Turtlebot3. As per the flowchart in Figure 2, the blob detection algorithm is used in this experiment. The result of testing shown in Fig. 3, the output image detect the circle form of yellow traffic light lamp.

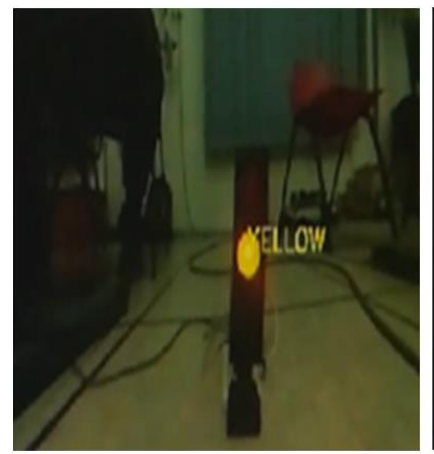

Before.

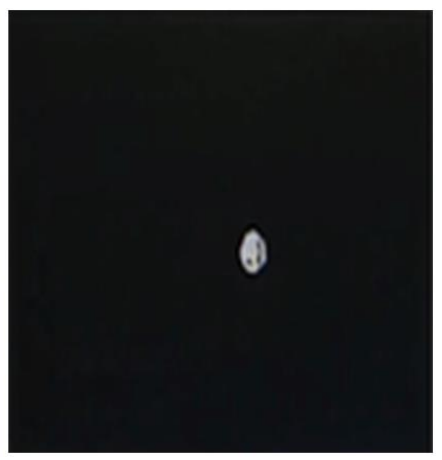

b. After
Fig3. Applying Blob Algorithm

In the preliminary stage, the traffic light colour detection are tested four times by using different threshold values that are adjusted manually. The threshold range values in Table 1-are generated by discarding the HSV boundary values that are too high or too low than the other HSV boundary value. The threshold range value below can be used as a reference to get the proper detection of the exact state of the active bulb.

A Linux operating system and OpenCV library are used as an application program interface (API) to perform the feature extraction on input images before the detection result can be generated. In this experiment, there are 114 input images or scene images that contain 209 traffic lights. Out of 209 traffic lights, there are 115 red light, 43 yellow light, and 51 green light. Throughout the experimental evaluation, the dataset information, as in Table II is used to determine the detection and accuracy rate.

Table-I: Threshold range value for traffic light detection

\begin{tabular}{lccc}
\hline & \multicolumn{3}{c}{ Threshold range value } \\
& Red & Yellow & Green \\
\hline Hue low & 0 & $16-26$ & $41-55$ \\
Hue high & $10-55$ & $35-64$ & $87-171$ \\
Saturation low & $30-92$ & $34-160$ & $86-165$ \\
Saturation high & $187-255$ & $84-255$ & $132-240$ \\
Lightness low & $48-162$ & $50-217$ & $30-89$ \\
Lightness high & 255 & $123-255$ & $132-255$ \\
& & & \\
\hline
\end{tabular}

Table- II: Dataset Information

\begin{tabular}{lcccc} 
& $\begin{array}{c}\text { Scene } \\
\text { images }\end{array}$ & $\begin{array}{c}\text { Red } \\
\text { light }\end{array}$ & $\begin{array}{c}\text { Yellow } \\
\text { light }\end{array}$ & $\begin{array}{c}\text { Green } \\
\text { light }\end{array}$ \\
\hline Daylight & 91 & 76 & 32 & 44 \\
Night & 23 & 39 & 11 & 7 \\
Total & 114 & 209 & 43 & 51 \\
\hline
\end{tabular}


There are four types of traffic lights tested in this system which are suspended type, horizontal and vertical side-pillar type and also centre pillar type (see Fig. 4). Besides, the experiments were carried out with images that consist of the different scenes image in complex intersections and environments. In this experiment, the resolution for all the scene images is below than $1024 \times 768$ with image size less than $250 \mathrm{kB}$.

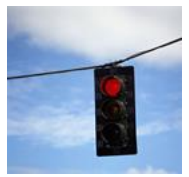

a. Suspended

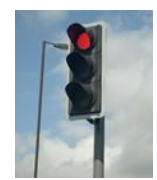

b. Centre pillar

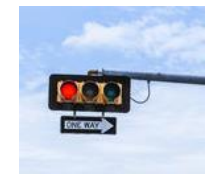

c. Side-pillar

(horizontal)

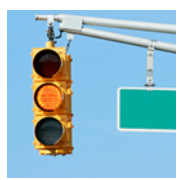

d.

Side-pillar

(vertical)
Fig. 4. Type of traffic lights

The initial experiment of detecting a traffic light colour was carried out on the prototype of traffic light during different times and weather conditions to reflect the diversity of driving and illumination conditions. The values of hue, saturation, and lightness to recognize the traffic light colour always change because the colour detection may be influenced by low contrast between the lights and the background. Since there is no fixed threshold value, then the threshold value should be set in a particular range. That is means that any values that can detect the traffic light colour in preliminary experiments are acceptable and can be used to identify the traffic light colour.

The extraction of colour information in this research is done by thresholding the traffic light colour pixels to get the candidate of the traffic lights. The BGR colour model is converted into HSV colour space since this colour model is based on human vision, and it can be used as a measure for distinguishing traffic light colours. It is because the colours of the traffic lights are not purely red, green and yellow but with slightly mixed with some other colours like red and yellow light contains some orange and the green light contains some blue.

Then, to ensure that only traffic light is being detected, it is very significant to eliminate the background because it is unnecessary noises. Therefore, the blob detection algorithm is used in a preliminary experiment and in evaluation experiments, the Hough circle transform are applied. Theoretically, Hough Circle Transform is only efficient when there are a high number of votes fall in the right bin so that the bin can be easily detected among the background noise. So, the bin must not be too small, or else some votes will fall in the neighbouring bins, thus reducing the visibility of the main bin. That is why in this traffic light system, there are a lot of traffic light images that cannot be detected because it is too small or too large than the set value.

It has been known that the Hough Circle Transform technique is mainly to detect the circle with the maximum vote. However, this method sometimes detects other circular objects instead of the traffic light. So, the Sobel filter is used to extract the edge of the detected turned-on light to eliminate the remaining noise. But, it is difficult to detect only the traffic light due to remained background noise. Hence, the
Gaussian blur is applied to reduce image noise and reduce detail in the background. The result from these techniques shows that the false positive in the output images is gradually decrease and not as critical as before (Fig. 5).
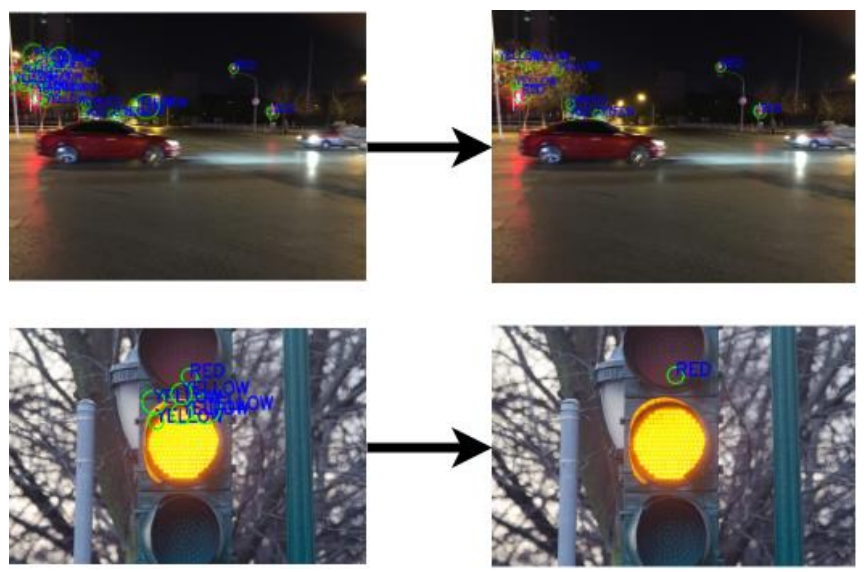

Fig. 5.Apply the Gaussian Blur And Sobel Filter. Before and After Results

Refer to Fig. 6, based on the experimental evaluation, the recognition accuracy is presented. The overall accuracy percentage achieve $75.59 \%$. The rate of accuracy at daylight and night-time is almost equal, hence the outcomes of the project are achievable.

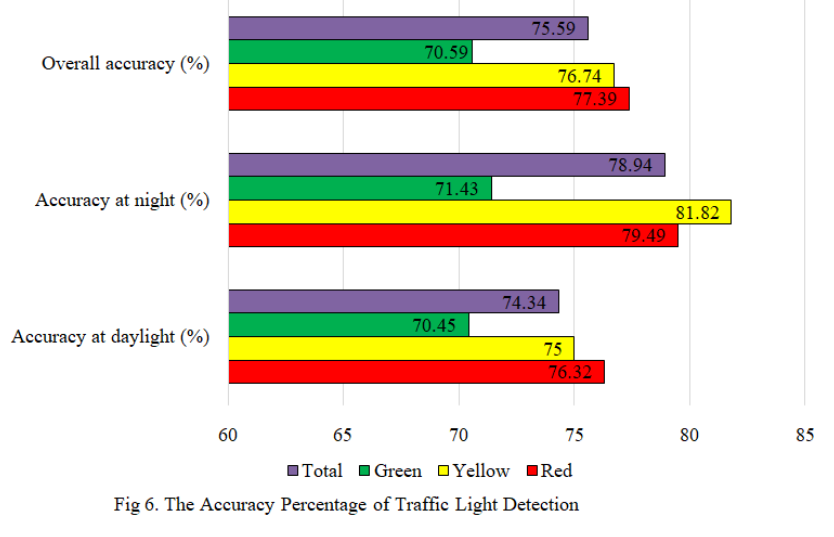

\section{CONCLUSION}

Based on the experimental result, the detection rate and accuracy percentage are reliable. It can extract traffic light in complex environments either at daylight or at night. Next, it also can detect the round-shape traffic light as well as an arrow-shaped traffic light. Out of 209 traffic light images, 158 images detected while 51 is unable to recognized and classified. So the overall accuracy percentage achieve based on the proposed algorithm is $75.59 \%$. This system is possible to be used in urban areas or complex environments, whether it is at night or day.

However, there are still imperfections in this proposed system. So, a lot of improvements need to be done to make it a perfect traffic light detection system. In future developments, this research can be improved by adding more filter or image processing techniques to increase the detection and accuracy rate even though it needs to work at all times of day under all possible lighting conditions.

Published By:

Blue Eyes Intelligence Engineering \& Sciences Publication 


\section{REFERENCES}

1. Shabadin, H. M. A case study of the prevalence and characteristics of red light runner in Malaysia. International Safer Roads Conference, 2014.

2. Radzak, M. Y. et al (2015). Study on traffic sign recognition. International Journal of Research Studies on Computer Science and Engineering (IJRSCSE), 2015 pp. 33-39.

3. Shioyma, T. W.Measurement of the length of pedestrian crossings and detection of traffic lights from image data. Measurement Science and Technology, 2002 pp. 1450-1457.

4. Bak, G.Traffic light recognition with HUV-histogram from daytime driving-view images. International Conference on Control, Automation and Systems. 2017

5. Zhenyang Wang, Z. D.Traffic light detection and tracking based on Euclidean Distance Transform and Local Contour Pattern. Chinese Intelligent Automation Conference: Intelligent Information Processing, 2013 pp. 623-631.

6. Yudin, D. S.Usage of fully convolutional network with clustering for traffic light detection. Meditteranean Conference on Embedded Computing (MECO). Budva, Montenegro. 2018

7. Omachi, M. O.Detection of traffic light using structural information. International Conference on Signal Processing Proceedings. Beijing, China. 2010

8. Sang-Hyuk Lee, J.-H. K.-J.Traffic light detection and recognition based on Haar-like features. International Conference on Electronics, Information, and Communication (ICEIC). Honolulu, HI, USA 2018

9. Wang, C. J. Robust and real-time traffic lights recognition in complex urban environments. International Journal of Computational Intelligence System, 2011 pp. 1383-1390.

\section{AUTHORS PROFILE}

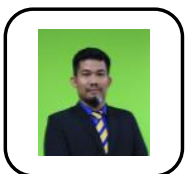

Mohamed Yusof Bin Radzak is a Senior Lecturer at Electrical, Electronics and Automation Section, Universiti Kuala Lumpur, Malaysian Spanish Institute. Graduated from Universiti Sains Malaysia and obtained B.Eng in Electrical and Electronics and Msc in Electrical and Electronics Engineering. Research work/interest on control and simulation, electric vehicles, and deep learning algorithm

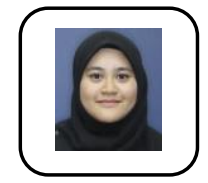

Nur Hanim Suraya Bt. Sabarudin pursuing her B. ,Eng Technology in Mechatronics (Automotive) from Universiti Kuala Lumpur, Malaysian Spanish Institute, Kulim Hi-Tech Park, Malaysia. Her research interest includes Machine Vision and Deep Learning Algorithm.

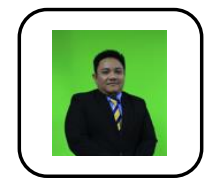

MohdFauzi Bin Alias is aLecturer at Electrical, Electronics and Automation Section, Universiti Kuala Lumpur, Malaysian Spanish Institute. Graduated from UniversitiSains Malaysia and obtainedB.Eng in Electrical and Electronics and Msc in Electrical and Electronics Engineering. Research work/interest on Algorithms, Software Engineering and Electronic Engineering 